\title{
Outage Analysis of Dual-Hop Transmission with Buffer Aided Amplify-and-Forward Relay
}

\author{
Guan-Xing Li, Chen Dong, Dong Liu*, Guangtao Li, and Yonghui Zhang
}

\begin{abstract}
A new packet-selection based transmission scheme is proposed on a dual-hop link with buffer aided amplify-andforward relay, where the relay with a buffer can record the channel information of source-relay hop and store the received packets. In order to satisfy the constraint of equivalent end-to-end SNR, the relay may pick up a packet in the buffer to transmit, with consideration of the channel statuses of both the source-relay hop and the relay-destination hop. Therefore, by adjusting the packet transmission order in the relay, the outage probability can be decreased in the proposed transmission scheme. Both theoretical analyses and simulation results show that the proposed scheme outperforms traditional ones by a significant margin of 2-4 dB gain in terms of outage probability.
\end{abstract}

\section{INTRODUCTION}

Nowadays, multihop wireless transmission based on relays has been increasingly investigated for its broader coverage and lower cost back-haul. The relays receive the signal from previous nodes, process and then forward it to subsequent nodes, which can benefit the wireless transmission system in terms of capacity, connectivity, adaptability, and ease of deployment [1-4]. Relays in multihop transmission can be classified into two types, one is non-regenerative relay that simply amplifies and forwards (AF) the signal, and the other is regenerative relay that performs detection then forwarding (DF). In this paper, we focus on dual-hop wireless transmission with an AF relay.

One problem of the conventional multihop system with AF relays, such as in $[5,6]$, is the high outage probability (OP) due to the fact that the outage occurs when any one of the channels in between the nodes is "bad." Indeed, the underlying reason is that the transmission works like a stream and thus will be restricted by the bottleneck. To alleviate this problem, we may break up the stream-like transmission into hop-by-hop, so as to achieve more reliable transmission. Therefore, multihop transmission with buffer aided relays has been proposed recently in [7-13].

In this paper, we proposed a dual-hop transmission scheme with buffer aided AF relay. The relay is assumed to be capable in recording the channel information of the source node $(\mathrm{SN})$

*Corresponding author. Dong Liu is with CAS Key Laboratory of Technology in Geo-spatial Information Processing and Application System, University of Science and Technology of China, Hefei 230027, China. Email: dongeliu@ustc.edu.cn.

Guan-Xing $\mathrm{Li}$ and Guangtao $\mathrm{Li}$ are with China Mobile Group Liaoning Ltd., Shenyang 110179, China. Email: \{liguanxing, liguangtao\}@ln.chinamobile.com.

Chen Dong is with School of ECS, University of Southampton, SO17 1BJ, UK. Email: cd2g09@ecs.soton.ac.uk.

Yonghui Zhang is with RF Micro Devices Co. Ltd., Beijing 100176, China Email: yhzhang@rfmd.com. to relay node (RN) hop. Moreover, the relay can store up to $B$ packets it received, where $B$ is the buffer size. Note that in an AF relay system, only the destination node (DN) decodes its received data, and the decoding is correct only when the equivalent end-to-end SNR is higher than a threshold. In order to satisfy the constraint of equivalent end-to-end SNR, the relay may adjust the transmission order of its stored packets according to the channel statuses. To be specific, the relay may pick up a packet in the buffer that underwent a "bad" channel in the SN-RN hop, when the RN-DN hop meets a "good" channel. On the contrary, a packet that underwent a "good" channel in the SN-RN hop may be sent by the relay when the RN-DN hop is "bad." In this manner, the outage probability of the entire transmission scheme can be decreased.

It is worthy to note that the buffer aided relays may incur transmission delay. However, the concepts of block delay and packet delay shall be distinguished [9]. Block delay is defined as the inverse of end-to-end throughput, and packet delay is the end-to-end delay of a specific packet. In our scheme, the block delay remains the same as in conventional schemes (i.e. without buffer), but the packet delay may be larger, and quite diverse for different packets. Thus, our proposed scheme is more suitable for non-real-time transmission.

This paper makes the following contributions. We proposed a new packet-selection based transmission scheme on a dualhop link with buffer aided AF relay. Compared with previous work, the new scheme realizes packet selection, rather than channel selection based on multihop diversity as in [7-9]. We have performed both theoretical analyses and simulation, and the results demonstrate the superior performance of our proposed scheme.

The remainder of this paper is organized as follows. Section II presents the system model of our proposed dualhop transmission scheme. Section III investigates the bound of outage probability, and describes a practical algorithm for simulation. Both theoretical and simulation results are provided in Section IV, followed by conclusions in Section V.

\section{System Model}

In this section, the system model is depicted into three parts. The system infrastructure is introduced first, followed by the transmission scheme and the fading channel model.

\section{A. Dual-Hop Link with Buffer Aided Relay}

The system model under consideration is a typical dualhop wireless link [6], as shown in Fig. 1. The dual-hop link consists of 3 nodes, an SN (node 0), an RN (node 1) and a DN 


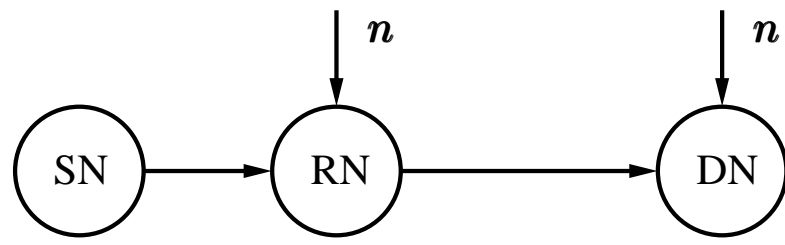

Fig. 1. System model of a dual-hop wireless link, where source node $S N$ sends messages to destination node $D N$ via intermediate relay node $R N, n$ stands for additive noise.

(node 2). The distance from the $\mathrm{SN}$ to the DN is normalized to $D_{S D}=1$. For simplicity, we assume that every hop spans the same distance, i.e. $D_{S R}=D_{R D}=d=1 / 2$. The SN transmits packets to the DN via the RN. At the RN, a buffer aided amplify-and-forward (AF) protocol is employed to relay the signal, which will be detailed in the next subsection.

For convenience, we denote the packet transmitted by node 0 as $x_{0}$ and its estimate at the DN by $x_{2}=\hat{x}_{0}$. We assume baseband QPSK modulation and that the signal is transmitted on the basis of time slots (TSs) having a duration of $T$ seconds. In addition to propagation pathloss, the channels of the 2 hops are assumed to experience independent block-based flat Extended Generalized- $\mathcal{K}$ (EGK) fading [14], which we will explain later. In the fading process, the complex-valued fading envelope of a hop remains constant within a TS, but is independently faded for different TSs. The pathloss is assumed to follow the exponential law of $d^{-\alpha}$, where $\alpha$ is the pathloss exponent, having a value between 2 and 6 , typically. We assume that the total energy per packet transmitted from the $\mathrm{SN}$ to the $\mathrm{DN}$ is $E_{p}=1$ unit, regardless of the number of hops. This one unit of energy is uniformly assigned to the 2 hops. Hence, the energy required for transmitting one bit over one hop is $1 / 2$ unit. Based on the above settings, the observation received by the DN can be expressed as

$$
\boldsymbol{y}=\sqrt{\frac{d}{2}} h_{2} \boldsymbol{G}\left(\sqrt{\frac{d^{-\alpha}}{2}} h_{1} \boldsymbol{x}_{0}+\boldsymbol{n}_{1}\right)+\boldsymbol{n}_{2},
$$

where $h_{1}, h_{2}$ represent the fading channel gain of the corresponding channels, and follow the Extended Generalized- $\mathcal{K}$ (EGK) distribution [14]. $\boldsymbol{n}_{1}, \boldsymbol{n}_{2}$ are the Gaussian noise added at RN and DN, respectively. The noise samples of $\boldsymbol{n}_{l}(l=1,2)$ obey the complex Gaussian distribution with zero mean and a fixed variance of $\sigma^{2}=1 /\left(2 \gamma_{p}\right)$ per dimension, where $\gamma_{p}$ denotes the received average SNR per packet if the SN directly sends signal to the DN over a single hop spanning a distance of $D_{S D}=1$ using one unit of energy.

Based on the above definitions and (1), the overall SNR at the DN can be written as

$$
\begin{aligned}
\gamma_{e q} & =\frac{\frac{d^{-\alpha}}{2}\left|h_{2}\right|^{2} G^{2} \frac{d^{-\alpha}}{2}\left|h_{1}\right|^{2}}{\frac{d^{-\alpha}}{2}\left|h_{2}\right|^{2} G^{2} N_{0}+N_{0}} \\
& =\frac{\gamma_{1} \gamma_{2}}{\gamma_{2}+\frac{1}{G^{2} N_{0}}},
\end{aligned}
$$

where $\gamma_{l}=\frac{d^{-\alpha}\left|h_{l}\right|^{2}}{2 N_{0}}, l=1,2$ are the instantaneous receiving SNR for the $l$-th hop, and $N_{0}$ is the power of noise. One possible choice of the relay gain, as given in $[6,15]$, reads

$$
G^{2}=\frac{1}{\left|h_{1}\right|^{2}+N_{0}}
$$

which leads to

$$
\gamma_{e q_{1}}=\frac{\gamma_{1} \gamma_{2}}{\gamma_{1}+\gamma_{2}+1}
$$

As a tight approximation, another choice suitable for analysis is

$$
G^{2}=\frac{1}{\left|h_{1}\right|^{2}}
$$

and leads to

$$
\gamma_{e q_{2}}=\frac{\gamma_{1} \gamma_{2}}{\gamma_{1}+\gamma_{2}}
$$

And let $\gamma_{\text {out }}$ be a threshold, if the $\gamma_{e q}$ is lower than $\gamma_{\text {out }}$, an outage occurs.

\section{B. Transmission Scheme}

Different from the traditional schemes, the buffer with size $B$ is added into the relay node. We consider a relatively large number of buffer size, e.g. 1024 packets or more, which is a realistic assumption nowadays. The transmission has two stages. In the first stage, $\mathrm{SN}$ transmits $B$ packets to $\mathrm{RN}$, and $\mathrm{RN}$ records the channel statuses that each packet undergoes. In the second stage, based on the instantaneous channel quality of the second hop, the RN picks up a packet for transmission, so that the packet can be successfully received at DN as the equivalent end-to-end SNR is higher than $\gamma_{\text {out }}$. The details of this protocol will be discussed in the next section.

\section{Channel Model}

Following [16], we use these notations in this paper: $\mathbf{E}\langle\bullet\rangle$ stands for expectation, $\Gamma(\bullet)$ is the gamma function [17] (8.310.1), $\Gamma(a, x, b, \beta) \triangleq \int_{x}^{\infty} t^{a-1} \exp \left(-t-b t^{-\beta}\right) d t$ is the extended incomplete Gamma function [18], $H_{m, n}^{p, q}[\bullet]$ is the Fox H-function [19] (1.2).

The channel model $[14,16,20]$ is,

$$
\begin{aligned}
f_{\gamma_{l}}(\gamma)= & \frac{\beta_{l}}{\Gamma\left(m_{l}\right) \Gamma\left(m_{s l}\right)}\left(\frac{b_{s l} b_{l}}{\bar{\gamma}_{l}}\right)^{m_{l} \beta_{l}} \gamma^{m_{l} \beta_{l}-1} \\
& \times \Gamma\left(m_{s l}-m_{l} \frac{\beta_{l}}{\beta_{s l}}, 0,\left(\frac{b_{s l} b_{l}}{\bar{\gamma}_{l}}\right)^{\beta_{l}} \gamma^{\beta_{l}}, \frac{\beta_{l}}{\beta_{s l}}\right)
\end{aligned}
$$

where $m_{l}\left(0.5<m_{l}<\infty\right)$ and $\beta_{l}\left(0<\beta_{l}<\infty\right)$ represent the fading severity and the fading shaping factor, respectively, $m_{s l}\left(0.5<m_{s l}<\infty\right)$ and $\beta_{s l}\left(0<\beta_{s l}<\infty\right)$ represent the shadowing severity and the shadowing shaping factor, respectively. In addition, $\bar{\gamma}_{l}=\mathbf{E}\left\langle\gamma_{l}\right\rangle$ denotes the average SNR of each hop. $b_{l}$ and $b_{s l}$ are defined as $b_{l}=\frac{\Gamma\left(m_{l}+\frac{1}{\beta_{l}}\right)}{\Gamma\left(m_{l}\right)}$, $b_{s l}=\frac{\Gamma\left(m_{s l}+\frac{1}{\beta_{s l}}\right)}{\Gamma\left(m_{s l}\right)}$. According to [19] (1.53), $f_{\gamma_{l}}(\gamma)$ can be expressed in terms of the Fox H-function as

$$
\begin{aligned}
f_{\left(\frac{b_{l} b_{s l} \gamma_{l}}{\gamma}\right)^{\beta_{l}}(\gamma)=} & \frac{1}{\Gamma\left(m_{l}\right) \Gamma\left(m_{s l}\right)} \times \\
& H_{0,2}^{2,0}\left[\gamma \mid\left(m_{l}-1,1\right),\left(m_{s l}-\frac{\beta_{l}}{\beta_{s l}}, \frac{\beta_{l}}{\beta_{s l}}\right)\right] .
\end{aligned}
$$

We use the term $f_{\left(\frac{b_{l} b_{s l} \gamma_{l}}{\bar{\gamma}}\right)}{ }^{\beta_{l}}(\gamma)$ as it describes the channel properties more specifically than $f_{\gamma_{l}}(\gamma)$. 
To summarize, we have made the following assumptions:

- The system is dual-hop link with amplify-and-forward relay.

- The RN knows the channel statuses of both the first hop channel and the second hop channel. The DN knows the channel status of the second hop channel.

- The RN can store at most $B$ packets and have recorded the channel qualities of each packet undergone.

- The fading processes of the two hops are independent. The fading of a given hop remains constant within a packet duration, but it is independent between different packets.

- The distribution of fading follows the Extended Generalized-J $\mathcal{K}$ (EGK) distribution.

\section{Outage AnAlysis And Simulation}

In this section, the idea of inverse channel matching is proposed for analysis. Then a practical algorithm is introduced for implementation. Finally, a comparison between conventional schemes and our proposed scheme is demonstrated to further distinguish the feature of the new idea.

Consider each packet that will experience two channels from $\mathrm{SN}$ to DN in Fig. 1. Here, a new idea about channel dividing and matching is provided to achieve a lower outage probability. As assumed, the relay has a buffer that can record the channel information for a fixed number of packets previously transmitted from $\mathrm{SN}$ to $\mathrm{RN}$. Then according to the recorded channel information of each packet and the current channel status of the second hop, an adaptive transmission order of the stored packets in $\mathrm{RN}$ will be decided. The key idea is to make the packets that have experienced a good channel in the first hop to go through a relatively bad channel in the second hop, and vice versa, so as to ensure a high successful transmission probability. The details of the idea are introduced next.

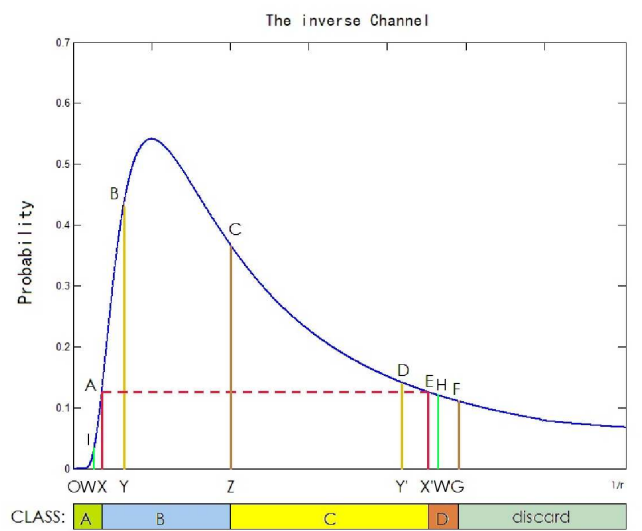

Fig. 2. The PDF of the inverse channel, where $x$ axis is the $1 / S N R$ of the inverse channel, hence the left terminal of $x$ axis represents the ideal channel that $1 / S N R$ is infinitesimal, and as $1 / S N R$ increases, the channel becomes worse; $y$ axis is the corresponding probability of each channel state.

\section{A. The Bound of Outage Probability with Inverse Channel Matching}

To derive the PDF of the inverse channel, let us start from the CDF of the Extended Generalized- $\mathcal{K}(\mathrm{EGK})$ channel,

$$
\begin{aligned}
& \int_{0}^{\gamma_{T}} f_{\left(\frac{b_{l} b_{s l} \gamma_{l}}{\bar{\gamma}}\right)^{\beta_{l}}}(\gamma) d \gamma \\
= & \int_{0}^{\infty} H_{1,1}^{1,0}\left[\frac{\gamma}{\gamma_{T}} \mid \begin{array}{c}
(1,1) \\
(0,1)
\end{array}\right] f_{\left(\frac{b_{l} b_{s l} \gamma_{l}}{\bar{\gamma}}\right)^{\beta_{l}}}(\gamma) d \gamma,
\end{aligned}
$$

where the definition of the Heaviside Theta function is [21] (14.05.02.0001.01) and its Fox H-function expression is given in [22] and [21] (14.05.26.0003.01). According to [19] (Section 2.3), we have

$$
\begin{aligned}
F_{\left(\frac{b_{l} b_{s l} \gamma_{l}}{\bar{\gamma}}\right)^{\beta_{l}}\left(\gamma_{T}\right)=} & \frac{1}{\Gamma\left(m_{l}\right) \Gamma\left(m_{s l}\right)} \times \\
& H_{1,3}^{2,1}\left[\gamma_{T} \mid\left(m_{l}, 1\right),\left(m_{s l}, \frac{\beta_{l}}{\beta_{s l}}\right),(0,1)\right]
\end{aligned}
$$

Substitute $\frac{1}{\gamma_{T}^{\prime}}$ for $\gamma_{T}$, the CDF of the inverse channel is

$$
\begin{aligned}
F_{\left(\frac{b_{l} b_{s l}}{\bar{\gamma} \gamma^{\prime}}\right) \beta_{l}\left(\gamma_{T}^{\prime}\right)=} & \frac{1}{\Gamma\left(m_{l}\right) \Gamma\left(m_{s l}\right)} \times \\
& H_{1,3}^{2,1}\left[\gamma_{T}^{\prime} \mid\left(m_{l}, 1\right),\left(m_{s l}, \frac{\beta_{l}}{\beta_{s l}}\right),(0,1)\right]
\end{aligned}
$$

By differentiating the CDF with respect to $\gamma_{T}^{\prime}$, after some deductions using [19] $(1.69,1.57)$, the PDF of the inverse channel is found as

$$
\begin{aligned}
& f_{\left(\frac{b_{l} b_{s l}}{\bar{\gamma} \gamma}\right)^{\beta_{l}}(\gamma)} \\
= & \frac{1}{\Gamma\left(m_{l}\right) \Gamma\left(m_{s l}\right)} \times \\
& H_{0,2}^{2,0}\left[\gamma \mid\left(m_{l}-1,1\right),\left(m_{s l}-\frac{\beta_{l}}{\beta_{s l}}, \frac{\beta_{l}}{\beta_{s l}}\right)\right] .
\end{aligned}
$$

Another solution is based on small quantity analysis leading to $f_{\frac{1}{\gamma}}(\gamma)=\frac{1}{\gamma^{2}} f_{\gamma}\left(\frac{1}{\gamma}\right)$. Substitute $h^{2}$ for $\gamma$, we also have the amplitude of the fading channel,

$$
\begin{aligned}
& f_{\frac{1}{\gamma}}(h) \\
= & \frac{2 \beta_{1}}{\Gamma\left(m_{l}\right) \Gamma\left(m_{s l}\right) h^{2 \beta_{l}+1}}\left(\frac{b_{l} b_{s l}}{\bar{\gamma}}\right)^{\beta_{l}} \times \\
& H_{0,2}^{2,0}\left[\frac{b_{s l} b_{l}}{h^{2} \bar{\gamma}} \mid\left(m_{l}-1,1\right),\left(m_{s l}-\frac{\beta_{l}}{\beta_{s l}}, \frac{\beta_{l}}{\beta_{s l}}\right)\right] .
\end{aligned}
$$

Fig. 2 shows the PDF of the inverse channel, where the point $G$ stands for the threshold of end-to-end SNR, i.e. $1 / \gamma_{\text {out }}$. As mentioned, when $1 / \gamma_{e q}>1 / \gamma_{\text {out }}$, the outage occurs. Therefore, according to (6), we have $1 / \gamma_{1}+1 / \gamma_{2}<G$ as the prerequisite of successful transmission. The lowest bound is

$$
1 / \gamma_{1}^{w}+1 / \gamma_{2}^{w}=1 / \gamma_{\text {out }}
$$

where $1 / \gamma_{1}^{w}$ and $1 / \gamma_{2}^{w}$ are denoted in Fig. 2 as points $X$ and $X^{\prime}$. Moreover, in Fig. 2, note that

$$
A X=E X^{\prime}
$$


which means the matching is not only in value but also in probability. These two points $X, X^{\prime}$ can be found based on (14) and (15).

After determining $X, X^{\prime}$, the point $Z$ is found out to satisfy $S_{A C Z X}=S_{C Z X^{\prime} E}$, where $S$ denotes the area, As shown in Fig. 2. According to the point $Z$, we may divide the channel status into four classes (A-D) plus discard. This division as well as channel matching are discussed as follows, please also refer to Fig. 2.

1) Channel matching of Class $B$ and Class $C$ : If one channel is in the region $X Z$, for example point $Y$, if set $Y^{\prime}$ according to (14), then the probability from $X$ to $Y$ is higher than that from $Y^{\prime}$ to $X^{\prime}$. This means the channel falls in class $\mathrm{B}$ more frequently than in class $\mathrm{C}$, thus a one-one matching is not feasible. To solve this issue, $Y^{\prime}$ can be moved leftward so that $O Y+O Y^{\prime}<O G$, so as to ensure that $S_{A X Y B}=$ $S_{D Y^{\prime} X^{\prime} E}$, i.e. $F_{\frac{1}{\gamma_{l}}}(Y)-F_{\frac{1}{\gamma_{l}}}(X)=F_{\frac{1}{\gamma_{l}}}\left(X^{\prime}\right)-F_{\frac{1}{\gamma_{l}}}\left(Y^{\prime}\right)$. Such $Y^{\prime}$ can be easily calculated.

2) Channel matching of Class $A$ and Class D: If the channel is in the region $O X$ (very good quality), it is easy to find the counterpart of it. For example, say the channel status is point $W$, and the counterpart is $W^{\prime}$ that lies in $X^{\prime} G$. This unknown $W^{\prime}$ can be found by $S_{X A I W}=S_{E X^{\prime} W^{\prime} H}$, i.e. $F_{\frac{1}{\gamma_{l}}}(X)-F_{\frac{1}{\gamma_{l}}}(W)=F_{\frac{1}{\gamma_{l}}}\left(W^{\prime}\right)-F_{\frac{1}{\gamma_{l}}}\left(X^{\prime}\right)$. Obviously $O W+\stackrel{\gamma_{l}}{O} O W^{\prime}<O^{\gamma_{l}} G$ is automatically satisfied, since the area $S_{O A X}$ is less than $S_{E F G X^{\prime}}$.

3) Outage probability: If it turns out that the equivalent end-to-end SNR is extremely low, it is possible that the point $Z$, found in the above process, may be on the left-hand side of the peak of the PDF. In this case, only channels in the area $O Z$ can find their counterparts in the area $Z G$. Therefore, the probability of successful transmission would be $2 \times S_{O Z C}$, i.e. the outage probability (OP) is $1-2 F_{\frac{1}{\gamma_{l}}}(Z)$.

\section{B. A Practical Algorithm at the Relay}

The above analysis provides a theoretical bound of the outage probability. In practice, we can use the following strategy at the relay. Based on the status of the second channel $\gamma_{2}$, the minimum acceptable SNR of the first channel is $\gamma_{1}=1 /\left(\frac{1}{\gamma_{\text {out }}}-\frac{1}{\gamma_{2}}\right)$. The relay can search the entire buffer to find the packet with the smallest SNR but still larger than $\gamma_{1}$. However, it should be noticed that practically we cannot make sure that every possible status of the second channel will find an exact matching status of the first channel. If the matching fails, then outage also occurs.

We may further conclude that the outage probability will decrease as the buffer size increases, in a practical relay, since larger buffer has more packets to search and therefore provides a better chance to find a matching. If the buffer size is large enough, the outage probability will approach to the theoretical bound.

\section{Conventional Scheme for Comparison}

We use the conventional scheme in [6] for comparitive study, which assumed that the transmission is over a Rayleigh fading channel and the end-to-end equivalent channel is (12) in that paper. Note that the outage probability of the conventional scheme serves as the benchmark.

\section{PERFORMANCE}

In this section, the performances of conventional transmission scheme and buffer aided relay scheme are provided for comparison. In the following, Conv. means conventional and C.O. (change order) represents the new scheme.

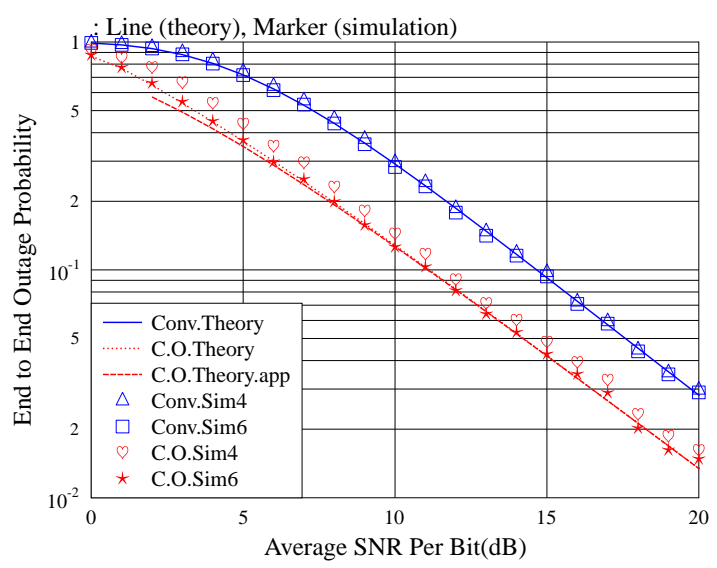

Fig. 3. SNR versus outage probability of different schemes over Rayleigh channels.

Fig. 3 presents the SNR from SN to DN per bit versus the outage probability. The Conv.Theory line shows the theoretical curve of the conventional scheme, according to [6] (12). The C.O.Theory line shows the theoretical bound as calculated by $1-\left(2 S_{O A X}+S_{X A B C D E X^{\prime}}\right)$ (refer to Fig. 2$)$, and the C.O.Theory.app line is an approximate bound calculated by $1-S_{O A B C D E F G}=F_{\gamma}\left(\gamma_{o u t}\right)$, which omits the search for $X, X^{\prime}$. The Conv.Sim 4 and C.O.Sim 4 are simulation results of the conventional scheme and the new scheme based on Eq. (4), whilst Conv.Sim6 and C.O.Sim6 are the corresponding results based on Eq. (6). A significant margin between the conventional scheme and the new scheme can be observed.

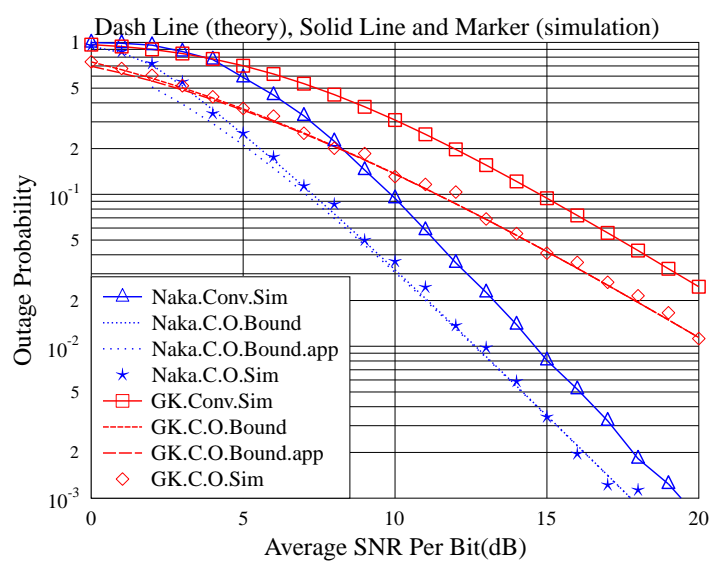

Fig. 4. SNR versus outage probability of different schemes over Nakagami$m$ and Generalized- $K$ channels. The $m$ value in Nakagami- $m$ is set as 2 and the $m, k$ values in Generalized- $K$ channel are set as $1.7,1.3$.

Fig. 4 presents the outage probability performances over Nakagami- $m$ and Generalized- $K$ channels, which are defined 
and described in [23,24]. The amplification gain $G$ is basd on Eq. (6). In Fig. 4, the Naka.Conv.Sim shows the simulation results of the conventional scheme over Nakagami$m$ channel, and Naka.C.O.Sim and Naka.C.O.Bound are the simulation and theoretical results of the new scheme. The Naka.C.O.Bound.app is an approximate bound as mentioned in Fig. 3. Similar terminologies are applied on the results over Generalized- $K$ channel. From this figure, we observe a significant performance gain of $2-4 \mathrm{~dB}$ in terms of outage probability versus SNR.

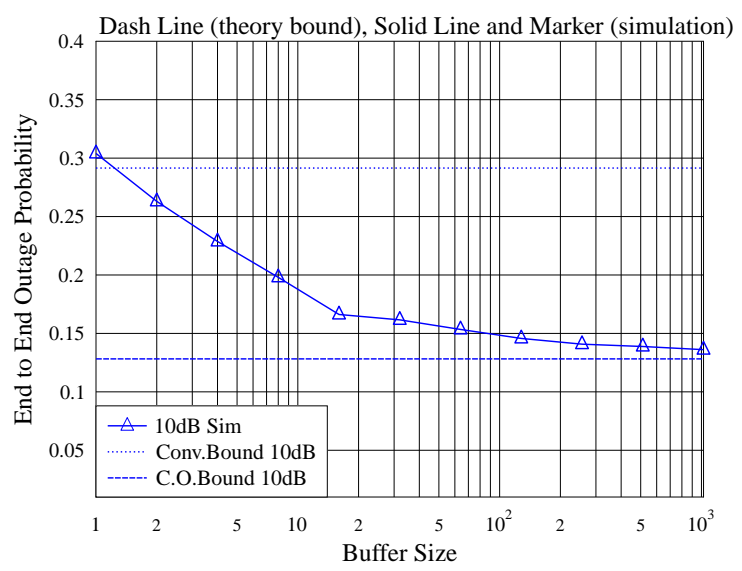

Fig. 5. Buffer size versus outage probability when SNR is $10 \mathrm{~dB}$. The outage probability decreases as the buffer size increases.

Fig. 5 shows the outage probability (OP) performance versus different choices of the buffer size at the relay, over the Rayleigh channel with SNR at $10 \mathrm{~dB}$. From this figure, the theoretical bound of OP of the conventional scheme is $29 \%$ (and simulated as buffer size equals 1 ); and the new scheme achieves a much lower OP at $12.9 \%$ (corresponding to infinite buffer size). The OP decreases by $1-12.9 / 29=55 \%$. Also, we observe that the OP will decrease more slowly when buffer size is large enough. In practice, the buffer size also controls the delay of packets, thus a medium buffer size is preferred to achieve a tradeoff between delay and OP. We would recommend buffer size equal to 16 or larger.

\section{CONCLUSIONS}

In this paper, a dual-hop transmission scheme with buffer aided AF relay is proposed. By adjusting the packet transmission order in the relay, the outage probability can be decreased in the proposed scheme. Both theoretical analyses and simulation results are provided on a dual-hop link over Extended Generalized- $\mathcal{K}$ (EGK) fading channel. Results show that the proposed scheme achieves $2-4 \mathrm{~dB}$ gain in terms of outage probability, compared with conventional schemes.

The proposed scheme can be extended to multihop transmission. Furthermore, we have adopted packet selection in the proposed scheme, rather than the channel selection based on multihop diversity as in [7-9], both techniques can be realized simultaneously in future work.

\section{REFERENCES}

[1] J. Boyer, D. D. Falconer, and H. Yanikomeroglu, "Multihop diversity in wireless relaying channels," IEEE Transactions on Communications, vol. 52, no. 10 , pp. $1820-1830$, Oct. 2004.
[2] A. Sendonaris, E. Erkip, and B. Aazhang, "User cooperation diversity. Part I. system description," IEEE Transactions on Communications, vol. 51, no. 11, pp. 1927 - 1938, Nov. 2003.

[3] — - "User cooperation diversity. Part II. implementation aspects and performance analysis," IEEE Transactions on Communications, vol. 51, no. 11 , pp. 1939 - 1948, Nov. 2003.

[4] J. N. Laneman, D. N. Tse, and G. W. Wornell, "Cooperative diversity in wireless networks: Efficient protocols and outage behavior," IEEE Transactions on Information Theory, vol. 50, no. 12, pp. 3062 - 3080, 2004.

[5] M. O. Hasna and M.-S. Alouini, "Outage probability of multihop transmission over Nakagami fading channels," IEEE Communications Letters, vol. 7, no. 5, pp. 216 - 218, May 2003.

[6] - "End-to-end performance of transmission systems with relays over Rayleigh-fading channels," IEEE Transactions on Wireless Communications, vol. 2, no. 6, pp. 1126 - 1131, Nov. 2003.

[7] C. Dong, L.-L. Yang, and L. Hanzo, "Multihop diversity for fading mitigation in multihop wireless networks," in IEEE Vehicular Technology Conference (VTC'11-Fall), San Francisco, CA, USA, Sept. 2011, pp. $1-5$.

[8] L.-L. Yang, C. Dong, and L. Hanzo, "Multihop diversity-a precious source of fading mitigation in multihop wireless networks," in IEEE Global Telecommunications Conference (Globecom'11), Houston, TX, USA, Dec. 2011, pp. 1-5.

[9] C. Dong, L.-L. Yang, and L. Hanzo, "Performance analysis of multihop diversity aided multi-hop links," IEEE Transactions on Vehicular Technology, vol. 61, no. 6, pp. $2504-2516,2012$.

[10] A. Ikhlef, D. S. Michalopoulos, and R. Schober, "Max-max relay selection for relays with buffers," IEEE Transactions on Wireless Communications, vol. 11, no. 3, pp. 1124 -1135, March 2012.

[11] N. Zlatanov and R. Schober, "Buffer-aided relaying with adaptive link selection-fixed and mixed rate transmission," IEEE Transactions on Information Theory, vol. 59, no. 5, pp. 2816-2840, 2013.

[12] N. Zlatanov, R. Schober, and P. Popovski, "Buffer-aided relaying with adaptive link selection," IEEE Journal on Selected Areas in Communications, vol. 31, no. 8, pp. 1530-1542, August 2013.

[13] N. Zlatanov, A. Ikhlef, T. Islam, and R. Schober, "Buffer-aided cooperative communications: opportunities and challenges," IEEE Communications Magazine, vol. 52, no. 4, pp. 146-153, April 2014.

[14] F. Yilmaz and M.-S. Alouini, "A unified MGF-based capacity analysis of diversity combiners over generalized fading channels," IEEE Transactions on Communications, vol. 60, no. 3, pp. 862 -875, Mar. 2012.

[15] J. N. Laneman and G. W. Wornell, "Energy-efficient antenna sharing and relaying for wireless networks," in IEEE Wireless Communications and Networking Confernce (WCNC'O0), San Francisco, CA, USA, vol. 1, Nov. 2000, pp. $7-12$ vol.1.

[16] K. P. Peppas, "A new formula for the average bit error probability of dual-hop amplify-and-forward relaying systems over generalized shadowed fading channels," IEEE Wireless Communications Letters, vol. 1 , no. 2, pp. $85-88$, april 2012.

[17] I. Gradshteyn and I. Ryzhik, Table of Integrals, Series, and Products, Seventh Edition. Elsevier Pte Ltd., 2007.

[18] M. Chaudhry and S. Zubair, "Extended incomplete gamma functions with applications," Journal of Mathematical Analysis and Applications, vol. 274, no. 2, pp. $725-745,2002$.

[19] A.M.Mathai, R. K. Saxena, and H. J.Haubold, The H-Function: Theory and Applications. Springer, Oct. 2010.

[20] F. Yilmaz and M.-S. Alouini, "A new simple model for composite fading channels: Second order statistics and channel capacity," in 7th International Symposium on Wireless Communication Systems (ISWCS'10), York, UK, Sept. 2010, pp. $676-680$.

[21] http://functions.wolfram.com/.

[22] V. Adamchic and O. Marichev, "The algorithm for calculating integrals of hypergeometric type functions and its realization in REDUCE system," in Proc. Int. Conf. on Symbolic and Algebraic Computation, 1990, pp. $212-224$.

[23] M. Nakagami, "The m-distribution, a general formula of intensity of rapid fading," Statistical Methods in Radio Wave Propagation: Proceedings of a Symposium, vol. 47, pp. 3-36, June 1958.

[24] P. S. Bithas, N. C. Sagias, P. T. Mathiopoulos, G. K. Karagiannidis, and A. A. Rontogiannis, "On the performance analysis of digital communications over generalized-K fading channels," IEEE Communications Letters, vol. 10, no. 5, pp. 353 - 355, may 2006. 